\author{
SERIES 'OCCUPATIONAL ASTHMA' \\ Edited by C. Mapp
}

\title{
The epidemiology of occupational asthma
}

\author{
W.S. Beckett
}

The epidemiology of occupational asthma. W.S. Beckett. OERS Journals Ltd 1994. ABSTRACT: With the accelerating pace of new knowledge about occupational asthma, systematic epidemiological approaches have yielded important new insights into its clinical characteristics. Such questions as "what proportion of all cases of asthma are caused by occupational exposures?" and "is occupational asthma incidence rising?" can now be answered.

Surveillance programmes estimate the number of exposed individuals and the number of incident cases, allowing us to perceive the magnitude of the health problem. Although occupational asthma is a relatively common illness, very little is known about quantitative exposure-response relationships. Epidemiological techniques can quantify the importance of predisposing factors, such as atopy or cigarette smoking, in determining individual risk factors for occupational asthma.

Still to be determined are the major, possibly genetic factors which make occupational asthma an "idiosyncratic" illness, affecting only a minority of those with equivalent exposures in the workplace. The goal of applying epidemiological techniques to the study of occupational asthma is ultimately to identify more effective means to prevent its occurrence.

Eur Respir J., 1994 7, 161-164.

The purpose of this review is to summarize current understanding of the epidemiology of occupational asthma, to describe the major designs of epidemiological studies, and to expose gaps in our knowledge that can be filled by further application of these techniques. As worldwide attention is focused on the increasing incidence of asthma, research has intensified to identify causative or contributory factors. Predisposing genetic factors, plus the inhalation of substances producing an inflammatory response, contribute to the clinical expression of asthma. The overall increase in cases of asthma cannot be explained by an increase in occupational asthma, because the age group with the greatest increase in cases is too young to be employed. Nonetheless, it is clear that some fraction of all new cases of asthma among working people is causally related to workplace exposures.

Because occupational asthma is, by its definition, a preventable disease, efforts to identify causative and contributory factors can lead directly to interventions that will reduce the burden of illness. The recognition and study of occupational asthma began with descriptive studies of single patients and small disease "clusters". These served to define the important clinical characteristics and to suggest pathophysiological mechanisms. Just as epidemiological approaches were helpful in bringing tuberculosis under control, epidemiological studies are now defining characteristics of occupational asthma as basic as how many people get it (incidence, prevalence), how much exposure is needed to cause disease (exposureresponse relationships), and why certain exposed individuals get it while others do not (associated risk factors). As this kind of information is determined for working groups,
Correspondence: W.S. Beckett, Occupational Medicine Program, Yale School of Medicine, 135 College St., 3rd floor, New Haven, CT 065010 USA.

Keywords: Asthma professionnel; isocyanates; occupational lung disease; respiratory disease epidemiology.

Received: July 161993

Accepted for publication September 121993

Supported in Part by Preventive Pulmonary Academic Award KO7-HL02316, Division of Lung Diseases, National Heart, Lung and Blood Institute. it will become possible to use ongoing surveillance as a means of determining the efficacy of measures taken to reduce the occurrence of occupational asthma.

\section{Case definition, incidence, prevalence, and case fatality rate}

Epidemiological studies are primarily concerned with quantifying the occurrence of disease, and with determining risk for disease. The basic unit of measurement is often a ratio, with cases of disease in the numerator, and the number of persons in the population at risk in the denominator. Epidemiological studies often compare the rates of disease between two groups (e.g. an exposed and unexposed group) [1]. A "case definition" is often made to make clear what the investigators consider to be a case. In some epidemiological studies, the case definition may deliberately emphasize standardization in criteria for diagnosis, to ensure uniformity when many physicians in different centres are applying the criteria. In other situations, the case definition may rely heavily on one or more diagnostic tests that are not widely available to clinicians, such as specific inhalation challenge. Depending on the objectives of the study, the case definition may be either more inclusive or more narrow. For example, to develop an occupational asthma surveillance system that could be uniformly applied to patients reported in several test states by many physicians, the United States National Institute for Occupational Safety and Health (NIOSH) developed a case definition requiring two major criteria plus one of four minor criteria (table 1).

An incidence rate; the number of new cases of disease 
occurring in a population of defined size over a unit period of time (e.g. new cases of asthma per 100,000 population per year) should be calculated, using only the population truly at risk for developing the disease (e.g. incidence of ovarian cancer in Belgium should not be calculated using the entire population, but rather the population of women, in the denominator). A calculation of occupational asthma incidence is provided by KESKINEN [2], who found 80 new occupational asthma cases reported in the Finnish registry of occupational diseases in 1976, and estimated a population of 492,500 individuals "at risk" by exposure to substances known to cause occupational asthma. The calculated incidence was thus 0.2 cases per 1,000 population at risk during the year of study.

One of very few prospective studies of occupational asthma, in which the presence or absence of asthma was established in the study group at the beginning of a period of observation of employees in an isocyanate manufacturing plant, found clinically important new cases of asthma in $4.3 \%$ of 168 exposed workers during a 5.5 yr observation period, indicating an incidence of approximately $0.8 \%$ (0.8 cases per 100 workers) per year [3].

The prevalence of occupational asthma is calculated as the number of cases at a specific point in time, divided by the population at risk because of exposure to known causative agents. For example, during an investigation of three cases of occupational asthma caused by isocyanate paints in an aircraft paint shop in Quebec, SEguin et al. [4] identified several further cases, and determined a prevalence of occupational asthma greater than $10 \%$ among the employees of the shop. Once prevalence of asthma is determined and an intervention has been made to prevent further cases, a second prevalence survey can then be performed to confirm whether the intervention has been successful. Note that both for incidence and prevalence, the ratio is valid only if the cases in the numerator have come from the population making up the denominator.

Although it is unknown what proportion of all cases of adult asthma are occupational in origin, estimates by knowledgeable observers have ranged from $2 \%$ (of all cases of asthma in the United States) [5] to $15 \%$ (of all adult male incident cases in Japan) [6]. These estimates

Table 1. - SENSOR (United States National Institute for Occupational Safety and Health) programme case definition of occupational asthma [10]

1. A physician diagnosis of asthma AND

2. An association between symptoms of asthma and work AND

3. Any one of the following:

a. Workplace exposure to an agent or process previously associated with occupational asthma OR

b. Significant work-related changes in forced expiratory volume in one second $\left(\mathrm{FEV}_{1}\right)$ or peak expiratory flow rate (PEFR) OR

c. Significant work-related changes in airway responsiveness as measured by nonspecific inhalation challenge

OR

d. Positive response to inhalation provocation testing with an agent to which the patient is exposed at work. are credible and widely quoted, but lack verification by population-based surveys. Based on the broad geographical range of case reports, occupational asthma occurs in most parts of the world, and in both agricultural and industrial areas. Approximately $6 \%$ of all asthma patients in a large university hospital respiratory clinic in Zambia were recently found to have occupational asthma [7]. Estimates of general prevalence based on hospital clinic populations must be interpreted with caution, because the referral pattern to a clinic may not represent the overall pattern of disease in the general population, and because occupational asthma occurs in clusters in certain industries, with marked geographical variation within a country. Such studies could overestimate or underestimate the true prevalence of the disease in the general population.

The importance of measuring the incidence or prevalence of occupational asthma, whether in a single workplace or a large state, is that an accurate assessment is needed both to determine its importance in prioritizing preventive measures, and because baseline data are needed to determine trends over time and the effectiveness of preventive measures. Surveillance is the ongoing process of counting cases of occupational asthma within a defined population, to establish and maintain awareness of the frequency of disease and to monitor control measures. One successful surveillance programme for occupational asthma has been the Surveillance of Work-related and Occupational Respiratory Disease (SWORD) programme in Britain, initiated by the British Thoracic Society about 4 yrs ago. This system uses a sampling strategy of reports from chest physicians and occupational physicians to report new cases of occupational lung disease. The SWORD programme estimated a total of 1,127 occupational asthma cases for the UK in $1992,30 \%$ of the total number of new occupational lung disease cases, the largest number of all categories of occupational lung disease [8]. Such surveillance systems may reveal important points of comparison and unanticipated new findings. For example, during the late 1980s, both the SWORD programme of the UK and the US SENSOR system (Sentinel Event Notification System for Occupational Risks) for the industrialized states of Michigan and Massachusetts found that the same class of industrial chemicals, the isocyanates, were the most frequently reported cause of occupational asthma [9-11]. Accurate interpretation of such epidemiological surveys requires awareness of potential causes of bias, (reasons why certain events may be reported more frequently than they actually occur). For example, isocyanates are widely known by chest physicians to cause asthma. This prior knowledge might lead physicians to inquire about, or recognize, isocyanates more frequently than other asthma causing substances.

\section{Natural history and case fatality rate}

With cessation of exposure to the sensitizing agent, many patients with occupational asthma have a complete remission of symptoms and require no further medication. Careful studies have demonstrated that other patients experience some improvement in their symptoms after removal from exposure, but continue to have symptomatic 
asthma over 5 yrs or more of follow-up. The follow-up study of 79 patients in the UK, referred to a tertiary hospital occupational asthma clinic with carefully documented occupational asthma, revealed the following outcomes over an average follow-up of 6 yrs: $90 \%$ had improvement in symptoms, $72 \%$ took medication, $40 \%$ reported limitation in activities, $33 \%$ were unemployed, and $10 \%$ required hospital admission [12].

The number of cases of disease with a fatal outcome, divided by the number of all cases, defines the case fatality rate. The death from status asthmaticus of a patient sensitized to toluene diisocyanate, reported by FABBRI et al. [13], has established that occupational asthma may be a fatal disease. Among the few long-term followup studies, fatal outcomes are rarely if ever described, suggesting that fatality is an exceptional outcome.

\section{Populations at risk and individual risk}

As noted above, the incidence and prevalence rates may be calculated only by using the "at risk" population in the denominator of the ratio. Because, in most cases, the threshold level of exposure capable of causing sensitization is not known, the "at risk" population is often approximated by the number of individuals with regular exposure to any level of the substance. A research group, attempting to define the population at risk for occupational asthma due to diisocyanates in the US, used survey data on the presence of isocyanates in selected workplaces throughout the country to estimate the total numbers of workers exposed. Based on visits to a sample of workplaces, it was estimated that for the years 1981-1983 in the US, 10,500 workers were potentially exposed to toluene diisocyanate (TDI), and 22,700 potentially exposed to diphenylmethane diisocyanate (MDI), both known to cause occupational asthma $[14,15]$.

When the true incidence of the disease is known, the individual risk for developing disease can be considered equivalent to the incidence. For example, in 1976, KESKINEN et al. [16] estimated the population at risk for occupational asthma in the Finnish food, beverage, and tobacco industries to be 65,300 , and found 20 cases of occupational asthma from the occupational disease registry for that year among employees in those industries. The risk for individuals in this population was thus $0.03 \%$, or 3 cases per 10,000 population.

\section{Analytic studies}

Specific inhalation challenge testing of a sensitized patient is often successful in demonstrating that a substance is capable of causing occupational asthma. The feasibility of directly demonstrating this in controlled challenges often makes analytical epidemiological studies unnecessary to demonstrate that a new asthma-causing agent has been found. There are other important uses, however, for cohort studies (in which subjects and controls are identified according to their exposure status and observed for the outcome of occupational asthma) and case-control studies (in which subjects and controls are identified by the presence or absence of occupational asthma, and compared by their exposure or risk factor status). Both types of studies are useful in testing whether a known exposure leads to disease. The case-control study may be more useful in circumstances where a specific causative agent among several substances has not been identified. Under these circumstances, the exposure histories of control subjects can be compared to cases of patients with disease, to determine what occupational exposures are more strongly associated with cases of asthma.

\section{Risk factors and attributable risk}

Just as a family history of heart disease, serum cholesterol level, hypertension and cigarette smoking are all independent risk factors for coronary heart disease, it is likely that there are multiple contributing factors (in addition to the sensitizing agent) in determining risk for occupational asthma. The presence of such risk factors would, thus, explain why some exposed workers develop clinical asthma, whilst others equally exposed do not. The casecontrol study design is particularly useful in analysing for such risk factors if the number of cases and controls is sufficient, and if there is a sufficient variation in the presence or absence of the risk factors to be tested.

Because the majority of cases of occupational asthma are mediated (at least in part) by a specific immunoglobulin $\mathrm{E}(\mathrm{IgE})$ produced in response to an inhaled antigenic substance, the atopic tendency to form such antibodies has been studied as a potential risk factor. To date, research indicates that an atopic tendency is a risk factor for asthma caused by certain agents that produce sensitization by specific IgE, but not for asthma caused by other agents. Occupational asthma caused by exposure to laboratory animals, one of the most widely appreciated causes of occupational asthma, has been found to be associated with atopy (in this case defined as a $3 \mathrm{~mm}$ wheal, at 10 min, to intradermal skin testing with one or more of three common allergens) [17]. This and other case-control studies [18, 19] have found a higher prevalence of atopy in exposed workers who developed asthma than in those who did not, indicating that atopy can be a risk factor for occupational asthma caused by certain substances. However, atopic status has not been shown to be related to the occurrence of asthma from other substances, such as the asthma caused by the plicatic acid of Western Red Cedar wood dust [20].

Cigarette smoking may also be a risk factor for occupational asthma from some causative agents, interacting with atopy to increase risk among exposed individuals. In a survey of workers exposed to tetrachlorophthalic anhydride (a substance known to cause occupational asthma), among those with specific IgE antibody to the chemical there was a greater proportion of smokers $(83 \%)$ compared to the group without specific antibody (48\% current smokers). The prevalence of specific antibody was highest in those who smoked and were atopic (positive skin tests) than among those who were smokers only or atopic only, indicating an interaction between cigarette smoking and atopy in the risk for developing specific IgE to tetrachlorophthalic anhydride. Here too, the risk factor of smoking applies to occupational asthma caused 
by some but not all causative agents. In a study of sawmill workers exposed to Thuja plicata (Western Red Cedar), smoking was not associated with increased risk for asthma [21]. It is interesting to note that pre-existing but subclinical nonspecific airway hyperresponsiveness has not been shown to be a risk factor for occupational asthma. In two prospective studies by BuTCHER et al. [3] and Chan-Yeung and DesjaRdins [22], subjects initially without nonspecific hyperresponsiveness were as likely to go on to develop occupational asthma as those with initial hyperresponsiveness.

The case-control study design can be particularly useful in determining a causative agent for asthma when there is no specific biological marker for sensitization (such as skin testing or specific inhalation challenge). A role for nonspecific or nonimmunological irritants in the causation of asthma has been proposed by KenNEDY [23]. The casecontrol design is also especially suited to studies of associated risk factors in diseases (like occupational asthma) which occur infrequently in the general population.

Much more research is required to clearly define the role of specific risk factors in occupational asthma. Progress is limited by the sporadic nature of occupational asthma, and by the relative difficulty in defining large groups for study. Available information strongly suggests that other factors, as yet unidentified, are important in the risk for occupational asthma. When larger numbers of those with disease are studied, it is possible to calculate the attributable risk or the proportion of cases of disease that would not occur in the absence of that risk factor. It seems likely that synergistic interactions among risk factors occur (causing greater risk for disease when two or more factors are present than the sum of the individual risks).

\section{Conclusion}

The ultimate purpose of applying these epidemiological techniques is to define as precisely as possible characteristics and causative factors for disease that may not be discernible from the study of individual patients. How much information about occupational asthma will epidemiological studies provide? Of course, it is already apparent in the case of occupational asthma that control of human exposures has the potential to prevent most cases, but the degree of control required to prevent all cases can be very difficult to achieve. We may reasonably expect to learn the relative importance of the major risk factors, the predictive value of their presence singly or in combination, and the efficacy in disease prevention of "modification" of risk factors. The insights gained from epidemiological study have proved highly effective in the prevention and control of many other occupational respiratory diseases, and are necessary to measure both the size of the problem and the progress being made against it.

\section{References}

1. Monson R. - In: Occupational Epidemiology. 2nd edn. Boca Raton, CRC Press, 1990; p. 27.

2. Keskinen H. - Epidemiology of occupational lung diseases: asthma and allergic alveolitis. In: Kerr JW, Ganderton MA, eds. Proceedings of XIth International
Conference of Allergology and Clinical Immunology. London, MacMillan, 1983; pp. 403-407.

3. Butcher BT, Jones RN, O'Neill CE, et al. - Longitudinal study of workers employed in the manufacture of toluene diisocyanate. Am Rev Respir Dis 1977; 116: 411-421.

4. Seguin P, Allard A, Cartier A, Malo J-L. - Prevalence of occupational asthma in spray painters exposed to several types of isocyanate, including polymethylene polyphenylisocyanate. J Occup Med 1987; 29: 340-344.

5. Brooks SM. - Bronchial asthma of occupational origin. Scand J Work Environ Health 1977; 3: 53.

6. Kobayashi S. - Different aspects of occupational asthma in Japan. In: CA Frazier, ed. Occupational Asthma. New York, Van Nostrand-Reinhold, 1980; pp. 229-244.

7. Syabbalo N. - Occupational asthma in a developing country. (Letter). Chest 1991; 99: 528.

8. McDonald JC, Meredith SK. - Would a SWORD-like surveillance scheme be useful and feasible in the USA? (Abstract). Am Rev Respir Dis 1993; 147: A903.

9. Meredith SK, Taylor VM, McDonald JC. - Occupational respiratory disease in the United Kingdom 1989: a report to the British Thoracic Society and the Society of Occupational Medicine by the SWORD project group. Br J Ind Med 1991; 48: 292-298.

10. Matte TD, Hoffman RE, Rosenman KD, Stanbury M. Surveillance of occupational asthma under the SENSOR model. Chest 1990 (Suppl.); 98: 173S-178S.

11. Wilrich K. - Personal communication. Massachusetts Occupational Health Surveillance Program, 1992.

12. Venables KM, Davison AG, Newman Taylor AJ. Consequences of occupational asthma. Respir Med 1989; 83: 437-440.

13. Fabbri LM, Danieli D, Crescioli S, et al. - Fatal asthma in a toluene diisocyanate-sensitized subject. Am Rev Respir Dis 1988; 137: 1494-1498.

14. National Occupational Exposure Survey (NOES). - Field Guidelines. US Dept of Health and Human Services, Pubic Health Service, Centers for Disease Control, National Institute for Occupational Safety and Health (NIOSH), DSHEFS, Cincinnati, Ohio 45226, March 1988.

15. Division of Hazard Evaluations and Field Studies, National Institute for Occupational Safety and Health, Cincinnati, Ohio. Unpublished data.

16. Keskinen H, Alanko K, Saarinen L. - Occupational asthma in Finland. Clin Allergy 1978; 8: 569-579.

17. Slovak AJM, Hill RN. - Does atopy have any predictive value for laboratory animal allergy? A comparison of different concepts of atopy. Br J Ind Med 1987; 44: 129-132.

18. Venables KM, Dally MB, Nunn AJ, et al. - Smoking and occupational allergy in workers in a platinum refinery. Br Med J 1989; 299: 939-942.

19. Burge PS, Perhs W, O'Brien IM, Hawkins R, Green M. - Occupational asthma in an electronics factory. Thorax 1979; 34: 13-18.

20. Chan-Yeung M. - Immunologic and non-immunologic mechanisms due to Western Red Cedar (Thuja plicata). J Allergy Clin Immunol 1982; 70: 32-37.

21. Chan-Yeung M, Lam S, Koerner S. - Clinical features and natural history of occupational asthma due to Western Red Cedar (Thuja plicata). Am J Med 1982; 72: 411-415.

22. Chan-Yeung M, Desjardins A. - Bronchial hyperresponsiveness and level of exposure in occupational asthma due to Western Red Cedar (Thuja plicata). Am Rev Respir Dis 1992; 146: 1606-1609.

23. Kennedy SM. - Acquired airway hyperresponsiveness from nonimmunogenic irritant exposure. Occupational Medicine: State of the Art Reviews. 1992; 7(2): 287-300. 